\title{
The curious arithmetic of optical vortices
}

\author{
Gabriel Molina-Terriza, Jaume Recolons, and Lluis Torner \\ Laboratory of Photonics, Department of Signal Theory and Communications, Universitat Politecnica da Catalunya, \\ Gran Capitan UPC-D3 Barcelona ES 08034, Spain
}

Received April 7, 2000

\begin{abstract}
The superposition of noncoaxial light beams containing screw wave-front dislocations is shown to create light patterns with a richer vortex content than that given by the arithmetic of the topological charges of the individual beams. We report the experimental observation of this phenomenon. (c) 2000 Optical Society of America

OCIS codes: $260.0260,120.4820,170.4520$.
\end{abstract}

Singular light beams that contain topological wavefront dislocations are ubiquitous entities that display fascinating properties with widespread, important applications. ${ }^{1,2}$ Screw dislocations, or vortices, are a common dislocation type.,4 They are spiral phase ramps around a singularity, where the phase of the wave is undefined and its amplitude vanishes. The order of the screw dislocation multiplied by its sign is referred to as the winding number, or the topological charge of the dislocation. Vortices appear spontaneously in several settings, including speckle fields, optical cavities, and doughnut laser modes. Otherwise, they can be readily generated, e.g., with phase masks, ${ }^{5}$ and nested in host beams. In the latter context a fundamental question arises about the vorticity of the light pattern generated by the superposition of individual vortex beams.

In the case of coaxial vortices nested in ideal plane waves, the winding number of the light beam generated by the multiplication of different waves is given by the arithmetic sum of the topological charges of the individual light beams. The same rule holds for the multiplication of finite beams. Clues that suggest the failure of such simple arithmetic rules in the sum of vortex beams are given by the superposition of vortices nested in coaxial beams with different shapes, where the number of existing vortices and their net topological charge is found to depend on the beam shapes and their relative widths and amplitudes, ${ }^{6}$ by the superposition of laser cavity modes, ${ }^{7}$ or by the explosions of perturbed higher-order dislocations. ${ }^{8}$ Our goal in this Letter is to show that the superposition of noncoaxial beams opens the door to a fascinating new range of possibilities. In particular, we reveal and experimentally observe that the superposition of noncoaxial vortex beams creates light patterns with a richer vortex content than that of the individual beams. The results fully reveal the curious arithmetic of screw wave-front dislocations and hold important practical implications for the implementation and operation of optical multitweezers with coherent light beams.

We address the linear superposition of two coherent light beams with nested vortices, with the general transverse form

$$
\begin{aligned}
S(x, y)= & {\left[X_{1}+i \operatorname{sgn}\left(m_{1}\right) Y_{1}\right]^{\left|m_{1}\right|} F_{1}\left(X_{1}, Y_{1}\right) } \\
& +\left[X_{2}+i \operatorname{sgn}\left(m_{2}\right) Y_{2}\right]^{\left|m_{2}\right|} F_{2}\left(X_{2}, Y_{2}\right),
\end{aligned}
$$

where $X_{\nu}=x-x_{\nu}$ and $Y_{\nu}=y-y_{\nu}$, with $\nu=1,2$. Here $\left(x_{\nu}, y_{\nu}\right)$ are the transverse locations of the vortices, $F_{\nu}$ are the shapes of the host beams, and $m_{\nu}$ are the topological charges of the nested vortices. The vortices are assumed to be located on the axis of each host beam. Superposition (1) should not be confused with the multiplication of fields that takes place when two vortices are nested in the same host beam ${ }^{3,4,9}$ or when vortices are created by parametric frequency mixing in nonlinear crystals. In such cases, under appropriate conditions the multiplication of fields yields the sum of the topological charges.

However, such is not the case for the superposition of vortices [Eq. (1)]. Instead, the number and the location of vortices that are present in the scalar field $S(x, y)$ depend on the shape and axial separation of the individual beams. In particular, even though $S(x, y)$ is constructed as the linear superposition of two vortices, in general it does not contain such a number of vortices. Similarly, the topological charge of the resulting field is not given by the sum of the charges of the individual vortices. A simple example that illustrates these points is the superposition of two identical and coaxial beams with a nested vortex of charge $m$ : The resulting field carries not a vortex with topological charge $2 m$ but rather a vortex with charge $m$. A similar insight is also provided by the superposition of the vortices nested in the mathematical host beams $F_{1}=F_{2}=$ constant. To be specific, let $m_{1}=m_{2}=1$. Then, one readily finds that $S(x, y)$ always contains only one single-charge vortex, regardless of the separation between the vortices carried by the individual beams. Such a single-charge vortex is located at $x=$ $\left(x_{1}+x_{2}\right) / 2, y=\left(y_{1}+y_{2}\right) / 2$.

In what follows, we examine the superposition of vortices nested in noncoaxial Gaussian beams. For simplicity we assume that diffraction is negligible, so the individual fields have the form $F_{\nu}\left(X_{\nu}, Y_{\nu}\right)=A_{\nu} \exp \left[-\left(X_{\nu}{ }^{2}+Y_{\nu}{ }^{2}\right) / w_{\nu}{ }^{2}\right]$. The two vortices are assumed to be located along the $x$ axis 
and separated symmetrically from the origin, so $x_{1}=x_{0}$ and $x_{2}=-x_{0}$. The simplest case corresponds to single-charge vortices nested in beams with equal width. The superposition of such beams can be written as

$$
\begin{aligned}
S(x, y)= & \left(x-x_{0}+i y\right) \exp \left[-\left(x-x_{0}\right)^{2}-y^{2}\right] \\
& +A_{2}\left(x+x_{0}+i y\right) \exp \left[-\left(x+x_{0}\right)^{2}-y^{2}\right],
\end{aligned}
$$

where we have set $w_{1}=w_{2}=1$ and $A_{1}=1$ so that the coordinates are scaled to the beam width and $A_{2}=$ $B \exp (i \phi)$ stands for the relative complex amplitude between the beams. When $A_{2}=1$, the locations of all vortices that are present in $S(x, y)$ can be readily calculated analytically. One finds that all the existing vortices are located on the $y=0$ axis, and their $x$ coordinates satisfy

$$
x / x_{0}=\tanh \left(2 x x_{0}\right) .
$$

When the initial separation between the individual beams reaches the critical value $x_{0}=x_{0 c}=1 / \sqrt{2}$, the number of existing vortices changes. Below $x_{0 c}$, the total field contains only one vortex. Such a vortex has a positive charge and is located at the origin. However, when the beams are separated by a critical distance, a vortex twin with a vanishing topological charge is nucleated at the origin. Beyond the critical separation, the two existing single-charge vortices with positive charge are expelled toward both sides of the origin, at which the negatively charged vortex remains. The exact evolution of the locations of all vortices as a function of $x_{0}$ is shown in Figs. 1(a) and 1(b). When the individual beams are well separated, so that $x_{0} \gg x_{0 c}$, one has $x \simeq \pm x_{0}$, consistent with intuitive expectations. However, the vortex with the negative charge is always present, so the net topological charge of the superposition of the two charge 1 vortices is always 1 . Yet when the individual vortices are separated by several beam widths, the negatively charged vortex hidden in the dark region surrounding the individual beams, where the field amplitude is vanishingly small. Thus the presence of the negatively charged vortex might be correspondingly difficult to detect.

When the two individual beams are not identical, the locations of the existing vortices can be calculated numerically. The complex amplitude $A_{2}$ controls the value of the critical separation $x_{0 c}$ at which the vortex twin is created as well as the subsequent locations of all the vortices. By and large, the value of the amplitude, $B=\left|A_{2}\right|$, modifies the locations of the vortices along the $x$ axis, whereas the phase $\phi$ makes the vortices move along the vertical $(y)$ axis; Figs. 1(c) and 1(d) illustrate a typical example. These plots show the location of the vortices generated by the superposition of two Gaussian beams of equal width as in Figs. 1(a) and 1(b), but with different amplitude and relative phase, as a function of the axial separation $x_{0}$. Note the connection between the behavior shown in Figs. 1(c) and 1(d) and the creation of vortex streets in walking parametric wave mixing. ${ }^{10}$ From an experimental viewpoint, the behavior of the vortex location as a function of the relative phase $\phi$ is most important, because according to Fig. 1(d) large values of $\phi$ make the negative vortex move away from the region of detectable light intensity. Note that when $B=1$ and $\phi= \pm \pi$, and hence $A_{2}=-1$, the vortex with the negative charge disappears at infinity in the transverse $x-y$ plane. The case $x_{0}=0$ corresponds to destructive interference of the beams.

Qualitatively similar results are obtained by the superposition of vortices with different or with multiple charges. An illustration of the former case is given by the superposition of a vortex of charge 2 and a vortex of charge 1 nested in Gaussian beams as in Eq. (2): One finds that the resulting field contains either two or four single-charge vortices, with a total charge equal to 2 . Note also that the superposition of two single-charge vortices as in Eq. (2) but with opposite sign produces two vortices located at $x=x_{0} \operatorname{coth}\left(2 x x_{0}\right)$; thus when $x_{0}=0$ the total beam contains no vortices but an edge dislocation instead. An example of the superposition of multicharged vortices is shown in Fig. 2, which corresponds to two noncoaxial Gaussian beams with a charge 3 vortex nested in both of them. When $x_{0}=0$ the superposition produces a vortex of charge 3 , but otherwise the light beam contains different patterns of single-charge vortices. The total charge of all vortices is always 3. When the two beams are well separated, three vortices with a positive charge accumulate near the location of each of the beams, and three negative vortices keep the total charge equal to 3 . With perfectly symmetric beams, one of the vortices with charge -1 remains at the center, as in Fig. 1.

To demonstrate experimentally the existence of the additional vortices we employed the setup shown in Fig. 3. A collimated light beam produced by a $\mathrm{He}-\mathrm{Ne}$ laser was split by beam splitter BS1. A single-charge screw wave-front dislocation was created and nested in one of the beams by use of computer-generated holograms. ${ }^{5}$ The other beam was used as a reference

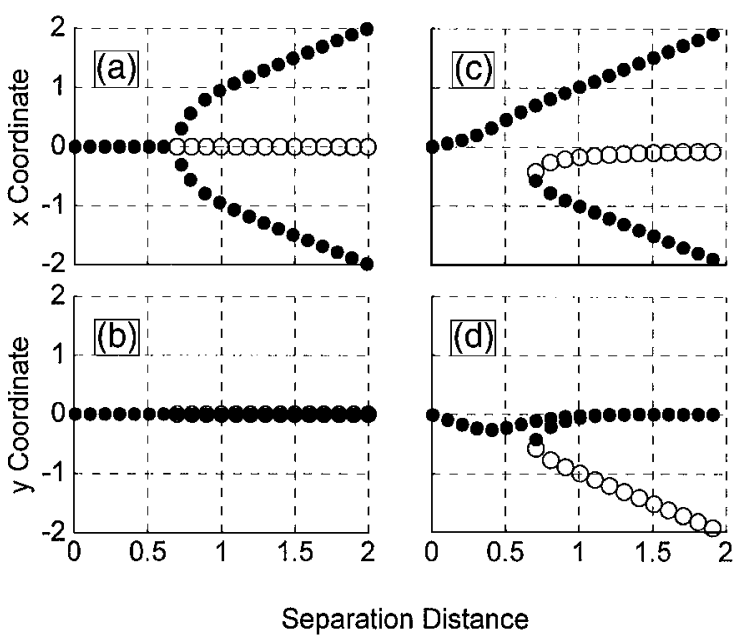

Fig. 1. Calculated locations of all the single-charge vortices that are present in the superposition $S$ as a function of the separation between the axes of the individual beams $\left(x_{0}\right)$. Filled circles, positive vortices, such as those nested on the individual beams; open circles, negative vortices. (a), (b) $B=1, \phi=0$; (c), (d) $B=0.6, \phi=\pi / 2$. 

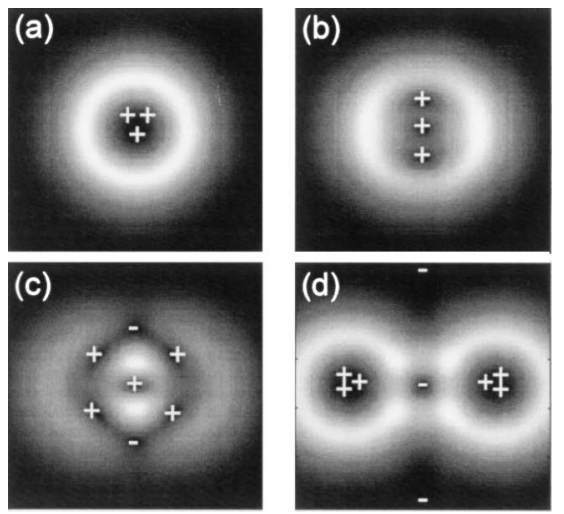

Fig. 2. Light pattern generated by the numerical superposition of two identical Gaussian beams with a charge 3 vortex nested in the center of each beam, for different separations between the beam axes: (a) $x_{0}=0.1$, (b) $x_{0}=0.4$, (c) $x_{0}=0.8$, (d) $x_{0}=1.75$. The plus and minus signs stand for the charge of the existing vortices. The symbols are to help the eye but do not necessarily indicate the exact locations of the vortices.

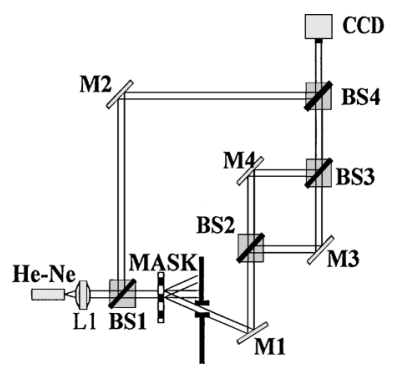

Fig. 3. Experimental setup: L1, lens 1; BS3, BS4, beam splitters; M1, mirror 1 . See text for other definitions.

wave. The beam containing the vortex was separated into two almost identical beams by beam splitter BS2. The path from BS2 to mirror M3 was used to separate the two beams transversely, and they were superposed again at beam splitter BS3. There must be an accurate match between mirrors M3 and M4 to make the beams collinear; otherwise, their interference, similar to that in Ref. 11, hides the negative vortex in the dark fringes. The intensity pattern of output light was analyzed by a CCD camera. We carefully scanned the wave front of the beam by changing the position of the reference wave, using mirror M2. Figure 4 shows a summary of our observations. Figures 4(a), 4(c), and 4(e) show the light intensity recorded for different beam separations; Figs. 4(b), 4(d), and 4(f) display the corresponding interferograms. The predicted vortex behavior, namely, the presence of the negatively charged vortex at the center of Fig. 4(d), is clearly visible.

In conclusion, we have shown that the superposition of noncoaxial light beams containing screw wave-front dislocations creates light patterns with a richer vortex content than that given by the arithmetic of the topological charges of the individual beams. We studied the particular example of linear superposition of vortices nested in Gaussian beams, but we believe that the results have implications for all systems that employ
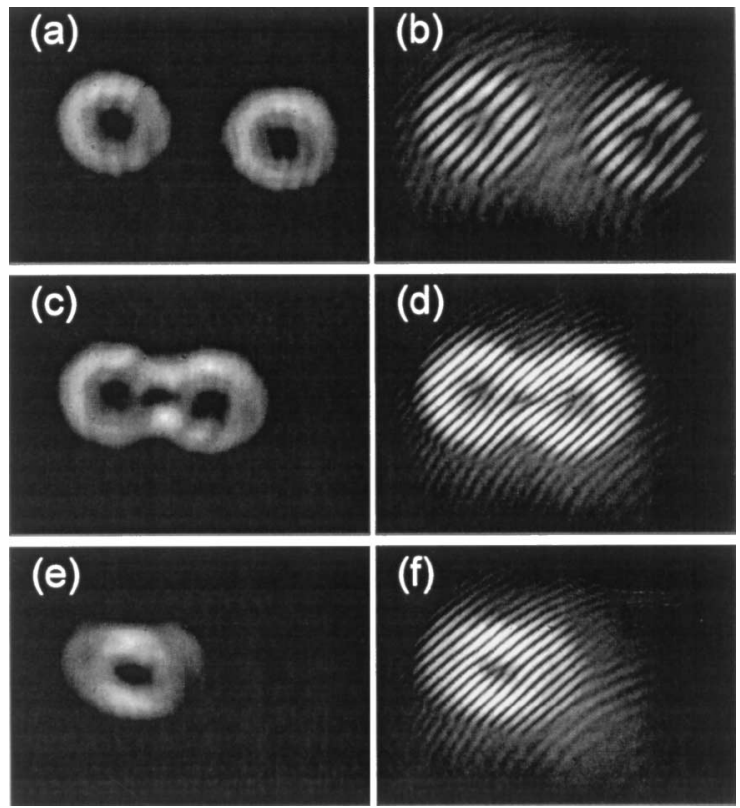

Fig. 4. (a), (c), (e) Observed light distribution and (b), (d), (f) interferograms produced by the superposition of two equal Gaussian beams with a single-charge vortex nested in the center of each beam. Axial separations between the beams: (a), (b) $x_{0} \sim 3.6 \mathrm{~mm}$, (c), (d) $x_{0} \sim 2 \mathrm{~mm}$, (e), (f) $x_{0} \sim 0.1 \mathrm{~mm}$. Waist of the input beams, $\sim 1 \mathrm{~mm}$.

superposition of coherent vortex entities, including collisions between vortex solitons, optical multitweezers, and vortex structures formed with Bose-Einstein condensates. ${ }^{12}$

This work was supported by the Generalitat de Catalunya. G. Molina-Terriza's e-mail address is molina@tsc.upc.es.

\section{References}

1. J. F. Nye and M. V. Berry, Proc. R. Soc. London Ser. A 336, 165 (1974).

2. A. Ashkin, Opt. Photon. News 10(5), 41 (1999); M. Padgett and L. Allen, Phys. World 10(9), 35 (1997).

3. G. Indebetouw, J. Mod. Opt. 40, 73 (1993).

4. D. Rozas, C. T. Law, and G. A. Swartzlander, J. Opt. Soc. Am. B 14, 3054 (1997).

5. N. R. Heckenberg, R. McDuff, C. P. Smith, and A. G. White, Opt. Lett. 17, 221 (1992).

6. M. S. Soskin, V. N. Gorshkov, M. V. Vasnetsov, J. T. Malos, and N. R. Heckenberg, Phys. Rev. A 56, 4064 (1997).

7. M. Brambilla, F. Battipede, L. A. Lugiato, V. Penna, F. Prati, C. Tamm, and C. O. Weiss, Phys. Rev. A 43, 5090 (1991); N. R. Heckenberg, M. Vaupel, J. T. Malos, and C. O. Weiss, Phys. Rev. A 54, 2369 (1996).

8. I. Freund, Opt. Commun. 159, 99 (1999).

9. B. Luther-Davies, R. Powles, and V. Tikhonenko, Opt. Lett. 22, 1816 (1994).

10. G. Molina-Terriza, L. Torner, and D. V. Petrov, Opt. Lett. 24, 899 (1999).

11. N. R. Heckenberg, R. McDuff, C. P. Smith, H. Rubinstein-Dunlop, and M. J. Wegener, Opt. Quantum Electron. 24, S951 (1992).

12. K. Burnett, M. Edwards, and C. W. Clark, Phys. Today 52(12), 37 (1999). 